\title{
RADEMACHER SERIES FROM ORLICZ TO THE PRESENT DAY
}

\author{
N. J. KALTON \\ Department of Mathematics, University of Missouri-Columbia, Columbia, MO 65211, U.S.A. \\ E-mail: nigel@math.missouri.edu
}

\begin{abstract}
We survey some questions on Rademacher series in both Banach and quasi-Banach spaces which have been the subject of extensive research from the time of Orlicz to the present day.
\end{abstract}

1. Introduction. Orlicz's work spanned over sixty years and it left a profound influence on many areas of mathematics. The result is that we all feel the influence of Orlicz on our own research in different ways.

In this article, which is based on my talk at the conference, I am going to consider the aspect of Orlicz's work which I feel had the most impact on my own research. This is the study of unconditional series and Rademacher series in Banach spaces. My aim is to show how Orlicz's original papers in the 1930's were instrumental in planting the seeds for some of the most profound developments in Banach space theory in the last fifty years. In particular I will emphasize the circle of ideas around type, cotype, Grothendieck's inequality and factorization theorems.

At the same time I want also to look in parallel at how the same ideas look in the setting of quasi-Banach spaces. Quasi-Banach spaces have been an interest of this author for over thirty years, and, although the theory has never enjoyed the popularity I would have hoped for, I think Orlicz, at least, would have been sympathetic to this line of research. Indeed the Banach school was clearly very interested in studying this wider class of spaces, and it is only the power of the Hahn-Banach theorem which has caused research since that time to emphasize the theory of Banach spaces over quasi-Banach spaces.

It is perhaps somewhat surprising that some of the main factorization results can be extended to quasi-Banach spaces. We will also see that some interesting additional phenomena can arise when we look at quasi-Banach spaces.

2000 Mathematics Subject Classification: 46B09, 46A16.

The author was supported by NSF grant DMS-0244515.

The paper is in final form and no version of it will be published elsewhere. 
I also give a discussion of some recent work of the author on decoupling of Rademacher series in the setting of quasi-Banach spaces. I will conclude with some thoughts on the possibility of a "non-locally convex" extension of Grothendieck's theorem.

2. Orlicz and the origins of type and cotype. Let $\left(\epsilon_{n}\right)_{n \in \mathbb{N}}$ denote a sequence of independent random variables with $\mathbb{P}\left(\epsilon_{n}= \pm 1\right)=\frac{1}{2}$. We call this a sequence of Rademachers.

In 1933, Orlicz ([32] and [33]) proved:

TheOREM 2.1. Suppose $\left(f_{n}\right)_{n \in \mathbb{N}}$ is a sequence in $L_{p}(0,1)$ where $1 \leq p<\infty$. If $\sum_{n=1}^{\infty} f_{n}$ converges unconditionally then

where $r=\max (p, 2)$.

$$
\sum_{n=1}^{\infty}\left\|f_{n}\right\|^{r}<\infty
$$

In these two papers, Orlicz became the first to apply Khintchine's inequality to problems in Banach space theory. At the time Khintchine's inequality was very new. It was proved by Khintchine only in 1925, and it should be remembered that information was not disseminated as quickly in those times as in the modern era. Orlicz saw that Khintchine's work had implications for Banach space theory, and in proving these results, he may be said to have introduced the probabilistic method into Banach space theory.

The study of Rademacher series has been particularly fruitful and it is also possible to argue that Orlicz in 1933 has essentially introduced the modern concept of cotype even though it was not to be formalized until many years later around 1972-1974 in work of Hoffmann-Jorgensen, Maurey and Pisier. The actual development of the precise notions of type and cotype is discussed in the recent article of Maurey in the Banach space Handbook [28].

Let us recall the definitions of type and cotype. A Banach space $X$ has (Rademacher) type $p$ where $1<p \leq 2$ if there is a constant $C$ such that for $x_{1}, \ldots, x_{n} \in X$,

$$
\left(\mathbb{E}\left\|\sum_{j=1}^{n} \epsilon_{j} x_{j}\right\|^{p}\right)^{\frac{1}{p}} \leq C\left(\sum_{j=1}^{n}\left\|x_{j}\right\|^{p}\right)^{\frac{1}{p}}
$$

and cotype $q$ where $2 \leq q<\infty$ if there is a constant $C$ such that, for $x_{1}, \ldots, x_{n} \in X$,

$$
\left(\sum_{j=1}^{n}\left\|x_{j}\right\|^{q}\right)^{\frac{1}{q}} \leq C\left(\mathbb{E}\left\|\sum_{j=1}^{n} \epsilon_{j} x_{j}\right\|^{q}\right)^{\frac{1}{q}} .
$$

One may argue that the following theorem is due to Orlicz for the case of cotype and Nordlander for the case of type [31].

Theorem 2.2. $L_{p}(0,1)$ has type $\min (p, 2)$ and cotype $\max (p, 2)$ when $1 \leq p<\infty$.

We should mention here the Kahane-Khintchine inequality which generalizes the Khintchine inequality to an arbitrary Banach space [13] :

Theorem 2.3. For any $0<p<q<\infty$ there is a constant $C=C(p, q)$ so that in any Banach space $X$ we have

when $x_{1}, \ldots, x_{n} \in X$.

$$
\left(\mathbb{E}\left\|\sum_{j=1}^{n} \epsilon_{j} x_{j}\right\|^{q}\right)^{\frac{1}{q}} \leq C\left(\mathbb{E}\left\|\sum_{j=1}^{n} \epsilon_{j} x_{j}\right\|^{p}\right)^{\frac{1}{p}}
$$


This theorem then expresses the equivalence of the quantities $\left(\mathbb{E}\left\|\sum_{j=1}^{n} \epsilon_{j} x_{j}\right\|^{p}\right)^{\frac{1}{p}}$ for any two choices of $p$ and allows us some flexibility in expressing the notions and cotype.

Let us conclude this section with an observation which combines the Kahane-Khintchine inequality and ideas implicit in Orlicz's 1933 paper:

TheOREM 2.4. For each $1 \leq p<\infty$ there is a constant $C$ so that

$$
C^{-1} \mathbb{E}\left\|\sum_{j=1}^{n} \epsilon_{j} f_{j}\right\|_{p} \leq\left\|\left(\sum_{j=1}^{n}\left|f_{j}\right|^{2}\right)^{\frac{1}{2}}\right\|_{p} \leq C \mathbb{E}\left\|\sum_{j=1}^{n} \epsilon_{j} f_{j}\right\|_{p}, \quad f_{1}, \ldots, f_{n} \in L_{p} .
$$

3. Factorization theorems and Grothendieck's inequality. Theorem 2.4 leads almost immediately to a vector-valued estimate for operators. A precursor of the following result was proved by Paley [34] and in its full force it is due to Marcinkiewicz and Zygmund [26] in 1939:

Theorem 3.1 (Marcinkiewicz-Zygmund). Suppose $1 \leq p, q<\infty$; then there is a constant $C=C(p, q)$ so that if $T: L_{p} \rightarrow L_{q}$ is a bounded operator then

$$
\left\|\left(\sum_{j=1}^{n}\left|T f_{j}\right|^{2}\right)^{\frac{1}{2}}\right\|_{q} \leq C\|T\|\left\|\left(\sum_{j=1}^{n}\left|f_{j}\right|^{2}\right)^{\frac{1}{2}}\right\|_{p}, \quad f_{1}, \ldots, f_{n} \in L_{p} .
$$

In Maurey's thesis [27] (published in 1974) vector-valued estimates such as (3.1) were used to obtain factorization theorems via a change of density. It follows that every operator $T: L_{p} \rightarrow L_{q}$ where $1 \leq q \leq 2 \leq p<\infty$ factors through a Hilbert space. Thus there is a direct line from Orlicz's early work to the study of operators which factor through Hilbert spaces. It was again Maurey who observed that an elegant theorem of Kwapień [23] could be recast as a factorization theorem for more general Banach spaces.

TheOREM 3.2. Suppose $X$ be a Banach space of type 2 and $Y$ is a Banach space of cotype 2. Then every operator $T: X \rightarrow Y$ factors through a Hilbert space, i.e. there is a Hilbert space $H$ and operators $S: X \rightarrow H$ and $R: H \rightarrow Y$ so that $T=R S$. In particular, a Banach space of type 2 and cotype 2 is isomorphic to a Hilbert space.

At this point we backtrack a little to recall the celebrated work of Grothendieck from 1956 [10] (note here that the journal volume is dated 1953 but the paper appeared in 1956). In fact for more than ten years this work was essentially overlooked prior to the appearance of the article of Lindenstrauss and Pełczyński in 1968 [25]. After 1968, the importance of Grothendieck's work was integrated rapidly into Banach space theory. Grothendieck's work revolved around a remarkable inequality, which can perhaps be seen best as a far-reaching extension of the Khintchine's inequality:

THEOREM 3.3 (Grothendieck's inequality). There is an absolute constant $K_{G}$ with the following property. If $\left(a_{j k}\right)_{j, k=1}^{n}$ is an arbitrary real matrix then for any elements $u_{1}, \ldots$, $u_{n}, v_{1}, \ldots, v_{n}$ in a real Hilbert space with

$$
\max _{1 \leq j \leq n}\left\|u_{j}\right\|, \max _{1 \leq k \leq n}\left\|v_{k}\right\| \leq 1
$$


we have

$$
\left|\sum_{j=1}^{n} \sum_{k=1}^{n} a_{j k}\left(u_{j}, v_{k}\right)\right| \leq K_{G} \max _{\substack{\left|\xi_{j}\right| \leq 1 \\\left|\eta_{k}\right| \leq 1}}\left|\sum_{j=1}^{n} \sum_{k=1}^{n} a_{j k} \xi_{j} \eta_{k}\right| .
$$

Grothendieck's inequality has numerous applications, but one is the remarkable result that the vector-valued inequality (3.1) of Marcinkiewicz and Zygmund (Theorem 3.1) holds equally when the domain is either a $C(K)$-space or $L_{\infty}$. Thus if $T: C(K) \rightarrow L_{q}$ is a bounded operator then

$$
\left\|\left(\sum_{j=1}^{n}\left|T f_{j}\right|^{2}\right)^{\frac{1}{2}}\right\|_{q} \leq K_{G}\|T\|\left\|\left(\sum_{j=1}^{n}\left|f_{j}\right|^{2}\right)^{\frac{1}{2}}\right\|_{C(K)}, \quad f_{1}, \ldots, f_{n} \in C(K) .
$$

This inequality leads in similar fashion to the fact that every operator $T: C(K) \rightarrow L_{q}$ where $q \leq 2$ must factor through a Hilbert space. Since $C(K)$ is very far from being of type 2 this is a surprising extension of Theorem 3.2. Thus Grothendieck's inequality injects something new and unexpected into factorization theory.

Why should Theorem 3.2 hold even for $C(K)$ spaces? This anomaly was resolved in 1980 by Pisier [37] with the following remarkable result:

TheOREm 3.4 (The abstract Grothendieck theorem). Let $X, Y$ be Banach spaces such that $X^{*}$ and $Y$ have cotype 2 and either $X$ or $Y$ has the approximation property. Then every operator $T: X \rightarrow Y$ factors through a Hilbert space.

We recall that a Banach space $X$ has the approximation property if for every compact subset $K$ of $X$ and every $\epsilon>0$ there is a finite-rank operator $T$ on $X$ with $\|T x-x\|<\epsilon$ for $x \in K$.

See [39] for a fuller discussion and a more precise statement in terms of approximable operators. Pisier [38] also constructed a Banach space $X$ with the property that $X$ and $X^{*}$ have cotype 2 but $X$ is not a Hilbert space. This space must fail the approximation property!

4. Factorization in quasi-Banach spaces. Let us now examine the same ideas in the setting of quasi-Banach spaces. We first recall that if $X$ is a quasi-Banach space then $X$ is said to be $p$-normable if for some constant $C$ we have

$$
\left\|\sum_{j=1}^{n} x_{j}\right\| \leq C\left(\sum_{j=1}^{n}\left\|x_{j}\right\|^{p}\right)^{\frac{1}{p}}, \quad x_{1}, \ldots, x_{n} \in X .
$$

A fundamental result of Aoki [2] and Rolewicz [42] asserts that every quasi-Banach space is $p$-normable for some $0<p \leq 1$. It follows in particular that we can, without loss of generality, assume that the quasi-norm is always a continuous function on $X$.

The notions of type and cotype may be defined equally in quasi-Banach spaces. A $p$-normable quasi-Banach space has type $p$ (when $0<p \leq 1$ ). In fact if $0<p<1$ then a quasi-Banach space has type $p$ if and only if it is $p$-normable $([15])$, but there are quasi-Banach spaces which have type 1 without being locally convex (for example the Ribe space, see [15]). On the other hand any quasi-Banach space of type $p>1$ is automatically 
isomorphic to a Banach space [14]. We also note that the Kahane-Khintchine inequality (Theorem 2.3) is equally valid for quasi-Banach spaces [15].

Much of our discussion of the spaces $L_{p}$ extends to the case when $0<p<1$ without alteration. The spaces $L_{p}$ when $0<p<1$ are of cotype 2 and type $p$ (which is equivalent to being $p$-normable of course). For example Theorem 2.4 holds equally if $0<p<1$ and this allows the extension of Theorem 3.1 to the full range $0<p, q<\infty$. We also note that Bennett [4] using results of Maurey [27] showed that (3.3) holds when $0<p<1$.

All of this suggests that the main results of the previous section all hold in quasiBanach spaces. In fact Theorem 3.2 certainly holds for $X, Y$ quasi-Banach spaces (although of course any quasi-Banach space of type 2 is a Banach space) but we do not know an explicit reference. On the other hand, Pisier's abstract Grothendieck theorem does have an appropriate formulation for quasi-Banach spaces, as was proved by the author and S. C. Tam in 1993 [21]:

TheOREM 4.1. Suppose $X$ and $Y$ are quasi-Banach spaces such that $X^{*}$ and $Y$ have cotype 2 and either $X$ or $Y$ has the bounded approximation property (BAP). Then every operator $T: X \rightarrow Y$ factors through a Hilbert space.

Here the dual of a quasi-Banach space $X$ is the Banach space of all continuous linear functionals $x^{*}$ with the norm $\left\|x^{*}\right\|=\sup _{\|x\| \leq 1}\left|x^{*}(x)\right| . X$ is said to have the bounded approximation property or (BAP) if there is a constant $C$ so that for every compact subset $K$ of $X$ and $\epsilon>0$ there is a finite-rank operator $T$ on $X$ so that $\|T\| \leq C$ and $\|T x-x\|<\epsilon$ for $x \in K$. Notice that the approximation assumption is stronger than in Theorem 3.4; it is unknown if the result can be improved in this respect.

A more precise statement, with the approximation assumption on the operator, is given in [21]. Note here that the assumption that $X^{*}$ has cotype two can be fulfilled vacuously if $X^{*}=\{0\}$ (e.g. when $X=L_{p}$ for $0<p<1$ ) but in that case the theorem is vacuous because $T=0$. The theorem really concerns finite-rank operators and the existence of non-trivial finite-rank operators which can only exist if $X^{*}$ does not reduce to zero. One immediate consequence is that if $X$ has (BAP) and both $X$ and $X^{*}$ have cotype two then $X$ is a Hilbert space. In the locally convex setting this result follows from Pisier's original abstract Grothendieck theorem under the weaker hypothesis that $X$ has the approximation property.

We recall that the Banach envelope $X_{c}$ of a quasi-Banach space $X$ is the Banach space obtained by completing the Hausdorff quotient of $X$ under the seminorm

$$
\|x\|_{c}=\sup \left\{\left|x^{*}(x)\right|:\left\|x^{*}\right\| \leq 1\right\} .
$$

Then an operator $T: X \rightarrow Y$ factors through a Banach space if and only if it factors through the natural map $X \rightarrow X_{c}$ or if and only if

$$
\|T x\| \leq C\|x\|_{c}, \quad x \in X,
$$

for some constant $C$. The following factorization theorem was also proved in [21].

TheOREM 4.2. Suppose $X$ and $Y$ are quasi-Banach spaces such that $X^{*}$ embeds into an $L_{1}$-space and $Y$ has nontrivial cotype. Suppose either $X$ or $Y$ has the bounded approximation property. Then every operator $T: X \rightarrow Y$ factors through a Banach space. 
It is not known whether this theorem can be improved to simply require that $X^{*}$ has some cotype (or even cotype 2). We may note also that the special case of Theorem 4.2 when $Y=L_{p}$ for $p>0$ was proved earlier (1986) in [17] and is a variation on the results of Bennett [4] and Maurey [27] cited above.

5. Special classes of quasi-Banach spaces. One of the themes of this article is that while Banach spaces may be somewhat bland, quasi-Banach spaces exhibit a rich variety in their behavior. While it might appear that all quasi-Banach spaces are uniformly bad, we may in fact isolate several different types of relatively good quasi-Banach spaces.

In [16] we introduced the notion of a natural quasi-Banach space. A quasi-Banach space $X$ is natural if it is isomorphic to a subspace of a quasi-Banach lattice $Y$ which is $p$-convex for some $p>0$, i.e. for some constant $C$ we have

$$
\left\|\left(\sum_{j=1}^{n}\left|y_{j}\right|^{p}\right)^{\frac{1}{p}}\right\| \leq C\left(\sum_{j=1}^{n}\left\|y_{j}\right\|^{p}\right)^{\frac{1}{p}}, \quad y_{1}, \ldots, y_{n} \in Y .
$$

The motivation for this definition is that most spaces that arise in analysis are natural (e.g. subspaces of Orlicz spaces, Lorentz spaces, etc.).

There is an alternative useful way to look at natural spaces. Let us say that a quasiBanach space $X$ is subordinate to a quasi-Banach space $Y$ if it is isomorphic to a subspace of an $\ell_{\infty}$-product of $Y$, or, alternatively, if there exists a constant $C$ so that for every $x \in X$ there exists an operator $T: X \rightarrow Y$ such that $\|T\| \leq 1$ and $\|x\| \leq C\|T x\|$. Then a separable space $X$ is natural if and only if it is subordinate to $L_{p}$ for some $p>0$ (see $[17])$.

If $X$ is a complex quasi-Banach space we say that $X$ is $A$-convex if it has an equivalent (continuous) quasi-norm which is plurisubharmonic, i.e.

$$
\|x\| \leq \int_{0}^{2 \pi}\left\|x+e^{i \theta} y\right\| \frac{d \theta}{2 \pi}, \quad x, y \in X .
$$

Since the quasi-norm on $L_{p}$ is plurisubharmonic every complex natural space is A-convex.

There are some basic examples to bear in mind here. The canonical example of a space which is not A-convex is the space $L_{p} / H_{p}$. More precisely we consider the space $L_{p}(\mathbb{T}, d \theta / 2 \pi)$ for $0<p<1$ and let $H_{p}$ be the subspace $\left[e^{i n \theta}: n \in \mathbb{N}\right]$. This example is due to Aleksandrov [1].

On the other hand consider the Schatten ideal $S_{p}$ (where $0<p<1$ ) of all compact operators $x$ on a separable Hilbert space satisfying $\sum s_{n}(x)^{p}<\infty$ where $s_{n}(x)$ is the $n$-th singular value of $x$. This space is A-convex but fails to be natural [18]; we will see the reason this space fails to be natural below in Section 8 .

6. Sidon sets and Rademacher series. Let $\left(f_{n}\right)_{n=1}^{\infty}$ and $\left(g_{n}\right)_{n=1}^{\infty}$ be a pair of sequences of real or complex-valued random variables. If $X$ is a quasi-Banach space we shall say that $\left(f_{n}\right)$ and $\left(g_{n}\right)$ are $(X, p)$-commensurate if there exists a constant $C$ so that

$$
C^{-1}\left(\mathbb{E}\left\|\sum_{j=1}^{n} f_{j} x_{j}\right\|^{p}\right)^{\frac{1}{p}} \leq\left(\mathbb{E}\left\|\sum_{j=1}^{n} g_{j} x_{j}\right\|^{p}\right)^{\frac{1}{p}} \leq C\left(\mathbb{E}\left\|\sum_{j=1}^{n} f_{j} x_{j}\right\|^{p}\right)^{\frac{1}{p}}, \quad x_{1}, \ldots, x_{n} \in X .
$$


Let us say that $\left(f_{n}\right)$ and $\left(g_{n}\right)$ are $X$-commensurate if there exists $C$ so that

$$
\mathbb{P}\left(\left\|\sum_{j=1}^{n} f_{j} x_{j}\right\| \geq t\right) \leq C \mathbb{P}\left(\left\|\sum_{j=1}^{n} g_{j} x_{j}\right\| \geq C^{-1} t\right), \quad x_{1}, \ldots, x_{n} \in X
$$

and

$$
\mathbb{P}\left(\left\|\sum_{j=1}^{n} g_{j} x_{j}\right\| \geq t\right) \leq C \mathbb{P}\left(\left\|\sum_{j=1}^{n} f_{j} x_{j}\right\| \geq C^{-1} t\right), \quad x_{1}, \ldots, x_{n} \in X .
$$

This terminology is a slight abuse of that introduced by Pełczyński in [35].

Let us suppose that $G$ is a compact abelian group and that $\mathbb{P}$ denotes normalized Haar measure on $G$. We recall that a sequence $\left(\chi_{n}\right)_{n=1}^{\infty}$ of characters on $G$ is called a Sidon set if for every choice of signs $\delta_{n}= \pm 1$ there is a finite Borel measure $\mu$ on $G$ with

$$
\int_{G} \bar{\chi}_{n} d \mu=\delta_{n}, \quad n=1,2, \ldots
$$

The sequence of Rademachers can be considered as a Sidon set of characters on the Cantor group $\{-1,1\}^{\mathbb{N}}$. Then Pisier in 1978 [36] (see also Pełczyński [35] for a generalization) showed that:

THEOREM 6.1. Let $\left(\chi_{n}\right)_{n=1}^{\infty}$ be a sequence of characters on $G$ and suppose $1 \leq p<\infty$. Then $\left(\chi_{n}\right)$ is $(X, p)$-commensurate with the Rademachers $\left(\epsilon_{n}\right)$ for every Banach space $X$ if and only if $\left(\chi_{n}\right)_{n=1}^{\infty}$ is a Sidon set.

From this Asmar and Montgomery-Smith [3] deduced that $\left(\chi_{n}\right)$ and $\left(\epsilon_{n}\right)$ are $X$-commensurate when $\left(\chi_{n}\right)$ is a Sidon set. They then raised the question of extending such results to quasi-Banach spaces. It turns out that Pisier's result does not extend to quasiBanach spaces, but there is a very satisfactory theorem. We recall that $\left(\chi_{n}\right)_{n=1}^{\infty}$ is called an $I_{0}$-set if for every sequence of signs $\delta_{n}= \pm 1$ there is a finite purely atomic Borel measure $\mu$ such that

$$
\int \bar{\chi}_{n} d \mu=\delta_{n}, \quad n=1,2, \ldots
$$

Equivalently given $\left(\delta_{n}\right)$ we can find $g_{n} \in G$ and complex $a_{n}$ with

$$
\sum_{n=1}^{\infty}\left|a_{n}\right|<\infty
$$

so that

$$
\sum_{k=1}^{\infty} a_{k} \chi_{n}\left(g_{k}\right)=\delta_{n}, \quad n=1,2, \ldots
$$

(This is not the original definition [11] but it is equivalent by a theorem of Kahane and Méla [12] and [29].)

The corresponding theorem to Theorem 6.1 is [19]:

THEOREM 6.2. Let $\left(\chi_{n}\right)_{n=1}^{\infty}$ be a sequence of characters on $G$ and suppose $0<p<\infty$. Then $\left(\chi_{n}\right)$ is $(X, p)$-commensurate with the Rademachers $\left(\epsilon_{n}\right)$ for every quasi-Banach space $X$ if and only if $\left(\chi_{n}\right)_{n=1}^{\infty}$ is an $I_{0}$-set.

Every Hadamard gap sequence in $\mathbb{N} \subset \mathbb{Z}$ is an $I_{0}$-set but not every Sidon set is an $I_{0}$-set. An example of a Sidon set in $\mathbb{Z}$ which is not an $I_{0}$-set is $\left\{3^{n}\right\}_{n=1}^{\infty} \cup\left\{3^{n}+n\right\}_{n=1}^{\infty}$. 
Nevertheless one can ask if for some quasi-Banach spaces Pisier's theorem remains true. The answer is yes:

THEOREM 6.3. Let $X$ be a quasi-Banach space which is subordinate to a space with nontrivial cotype (e.g. $X$ is natural). Then for any $0<p<\infty$ every Sidon set is $(X, p)$ commensurate with the Rademachers.

For natural spaces this was proved in [19]; the more general statement (which applies for example to the Schatten ideals $S_{p}$ ) is given in [21]. It is in fact a direct consequence of Theorem 4.2 above.

7. Decoupling. We now turn to the topic of decoupling. Let $\left(\epsilon_{n}\right)_{n=1}^{\infty}$ and $\left(\epsilon_{n}^{\prime}\right)_{n=1}^{\infty}$ be two mutually independent sequences of Rademachers. If we consider the sets $\left(\epsilon_{j} \epsilon_{k}\right)_{j<k}$ and $\left(\epsilon_{j}^{\prime} \epsilon_{k}\right)_{j, k}$ as characters on the Cantor group (or its square) they do not form Sidon sets. However it follows quite easily from Theorem 3.1 by a Fubini argument that for $0<p<\infty$ there is a constant $C=C(p)$ so that if $\left(f_{j k}\right)_{j, k=1}^{n}$ is an $L_{p}$-valued matrix then

$$
C^{-1} \mathbb{E}\left\|\sum_{j, k} \epsilon_{j}^{\prime} \epsilon_{k} f_{j k}\right\|_{p} \leq\left\|\left(\sum_{j, k}\left|f_{j k}\right|^{2}\right)^{\frac{1}{2}}\right\|_{p} \leq C \mathbb{E}\left\|\sum_{j, k} \epsilon_{j}^{\prime} \epsilon_{k} f_{j k}\right\|_{p} .
$$

Following Pisier [36] we say that a quasi-Banach space $X$ has property $(\alpha)$ if there is a constant $C$ so that for every $X$-valued matrix $\left(x_{j k}\right)_{j, k=1}^{n}$ and every scalar matrix $\left(a_{j k}\right)_{j, k=1}^{n}$ we have

$$
\mathbb{E}\left\|\sum_{j, k} a_{j k} \epsilon_{j}^{\prime} \epsilon_{k} x_{j k}\right\| \leq C \max \left|a_{j k}\right| \mathbb{E}\left\|\sum_{j, k} \epsilon_{j}^{\prime} \epsilon_{k} x_{j k}\right\|
$$

The above remarks show that $L_{p}$ has property $(\alpha)$ when $0<p<\infty$. This is equivalent to the statement that $\left(\epsilon_{j}^{\prime} \epsilon_{k}\right)$ is $\left(L_{p}, q\right)$-commensurate with the Rademachers for any $0<$ $p, q<\infty$ (using also a Kahane-Khintchine inequality).

Of course not every Banach space has property $(\alpha)$; it fails for the Schatten ideals $S_{p}$ when $1 \leq p<\infty$. It is also not in general true that the sequences $\left(\epsilon_{j} \epsilon_{k}\right)_{j<k}$ and $\left(\epsilon_{j}^{\prime} \epsilon_{k}\right)_{j<k}$ are $(X, p)$-commensurate for any choice of $p$; this fails for the trace-class $S_{1}$. However the simplest form of a decoupling theorem asserts that $\left(\epsilon_{j} \epsilon_{k}\right)_{j<k}$ is $(X, p)$-commensurate with $\left(\frac{1}{2}\left(\epsilon_{j}^{\prime} \epsilon_{k}+\epsilon_{k}^{\prime} \epsilon_{j}\right)\right)_{j<k}$ for every $p$ and every Banach space $X$ (indeed they are even $X$-commensurate).

Let us therefore define a quasi-Banach space $X$ to have the decoupling property if there is a constant $C$ so that if $\left(x_{j k}\right)_{j, k=1}^{n}$ is a symmetric $X$-valued matrix with zero diagonal then

$$
C^{-1} \mathbb{E}\left\|\sum_{j, k} \epsilon_{j} \epsilon_{k} x_{j k}\right\| \leq \mathbb{E}\left\|\sum_{j, k} \epsilon_{j}^{\prime} \epsilon_{k} x_{j k}\right\| \leq C \mathbb{E}\left\|\sum_{j, k} \epsilon_{j} \epsilon_{k} x_{j k}\right\| .
$$

Every Banach space has the decoupling property; this is a special case of a result of Kwapień [24] (see also [6]). In fact Banach spaces satisfy much more general decoupling results; we refer to the work of de la Peña and Montgomery-Smith [8] and [9] or the book of de la Peña and Giné [7] for further details. It turns out that essentially the same results can be proved in any quasi-Banach space with the decoupling property as shown in [20]. 
The question thus arises as to which spaces have the decoupling property. It is not too hard to show that $L_{p}$ for $0<p<1$ has the decoupling property; this can be shown by using classical work of Bonami on multiple Rademacher series [5] but also follows from the following theorem [20]:

Theorem 7.1. Property $(\alpha)$ implies the decoupling property.

Based on the case of $L_{p}$ it is easy to show that:

THEOREM 7.2. Every natural space has the decoupling property.

It may also be shown that the space $L_{p} / H_{p}$ (which is not A-convex) also has property $(\alpha)$ and hence the decoupling property [20]. This uses ideas of Pisier [41] and Kislyakov $[22]$.

On the other hand the Schatten ideals $S_{p}$ for $0<p<1$ fail decoupling; this will be clear from the next section.

8. Bilinear maps and Grothendieck's theorem revisited. Let us now give an application of the decoupling property (see [18] and [20]).

THEOREM 8.1. Let $X$ and $Y$ be Banach spaces of type 2 and suppose $Z$ is a quasi-Banach space with the decoupling property. Let $B: X \times Y \rightarrow Z$ be a bounded bilinear map. Then there is a constant $C$ so that

$$
\left\|\sum_{j=1}^{n} B\left(x_{j}, y_{j}\right)\right\| \leq C \sum_{j=1}^{n}\left\|x_{j}\right\|\left\|y_{j}\right\|, \quad x_{1}, \ldots, x_{n} \in X, y_{1}, \ldots, y_{n} \in Y .
$$

Thus $B$ factors through a Banach space, i.e. there is a Banach space $E$, a bounded bilinear map $B_{0}: X \times Y \rightarrow E$ and a linear operator $S: E \rightarrow Z$ so that $B=T B_{0}$.

If we consider the canonical bilinear map $B: \ell_{2} \times \ell_{2} \rightarrow S_{p}$, where $0<p<1$, given by $B(x, y)=x \otimes y$ then it is clear that (8.1) does not hold and $B$ does not factorize through a Banach space. Thus $S_{p}$ fails the decoupling property and is not natural.

If we consider Theorem 8.1 as a factorization theorem it is natural to consider it as an analogue of the Kwapień-Maurey theorem (Theorem 3.2) and ask if there is a similar abstract Grothendieck type theorem as in Theorem 3.4 or Theorem 4.1. Thus we would hope to replace the hypothesis in Theorem 8.1 that $X$ and $Y$ have type 2 with the hypothesis that $X$ and $Y$ have the (BAP) and $X^{*}$ and $Y^{*}$ have cotype 2.

In the special case when $X=Y=c_{0}$ and $Z=L_{p}$ for $0<p<1$ this reduces to a question about a generalized form of Grothendieck's inequality. Let us state this formally: Problem. Suppose $0<p<1$; is there a constant $K=K(p)$ so that if $\left(f_{j k}\right)_{j, k=1}^{n}$ is an $L_{p}$-valued matrix such that

then

$$
\sup _{\delta_{j}= \pm 1} \sup _{\delta_{k}^{\prime}= \pm 1}\left\|\sum_{j=1}^{n} \sum_{k=1}^{n} \delta_{j} \delta_{k}^{\prime} f_{j k}\right\|_{p} \leq 1
$$

$$
\left\|\sum_{j=1}^{n} \sum_{k=1}^{n}\left(u_{j}, v_{k}\right) f_{j k}\right\|_{p} \leq K
$$

where $u_{1}, \ldots, u_{n}, v_{1}, \ldots, v_{n} \in \ell_{2}$ with $\max \left\|u_{j}\right\|, \max \left\|v_{k}\right\| \leq 1$ ? 
It is possible to prove some results which are quite close to our conjecture. For example the following result would follow from the Nikishin factorization theorem [30] or [40] if we could achieve a factorization through a Banach space. We however can derive it directly from Theorem 4.1 above (see [20]):

TheOREM 8.2. Suppose $0<p<1$ and that $X$ and $Y$ are quasi-Banach spaces with the bounded approximation property and such that $X^{*}$ and $Y^{*}$ have cotype two. Then there is a constant $C=C(X, Y, p)$ so that if $B: X \times Y \rightarrow L_{p}(\Omega, \mu)$ is a bounded bilinear map then there is function $w \in L_{1}(\mu)$ with $w \geq 0$ a.e., $\int w d \mu=1,\{w=0\} \subset\{|B(x, y)|=0\}$ a.e. for all $x \in X$ and $y \in Y$ and

$$
\left\|w^{-\frac{1}{p}} B(x, y)\right\|_{L_{1, \infty}(w d \mu)} \leq C\|B\|\|x\|\|y\| .
$$

Finally we can deduce from Theorem 8.2 a result which says that (8.1) almost holds under these hypotheses:

COROLlary 8.3. Suppose that $X$ and $Y$ are Banach spaces with the bounded approximation property and such that $X^{*}$ and $Y^{*}$ have cotype two and $Z$ is a natural quasi-Banach space. Then there is constant $C=C(X, Y, Z)$ so that if $B: X \times Y \rightarrow Z$ is a bounded bilinear map and $x_{1}, \ldots, x_{n} \in X, y_{1}, \ldots, y_{n} \in Y$ are such that $\sum_{j=1}^{n}\left\|x_{j}\right\|\left\|y_{j}\right\|=1$ then

$$
\left\|\sum_{j=1}^{n} B\left(x_{j}, y_{j}\right)\right\| \leq C\|B\|\left(1+\sum_{j=1}^{n}\left\|x_{j}\right\|\left\|y_{j}\right\| \log \frac{1}{\left\|x_{j}\right\|\left\|y_{j}\right\|}\right) \text {. }
$$

\section{References}

[1] A. B. Aleksandrov, Essays on nonlocally convex Hardy classes, in: Complex Analysis and Spectral Theory (Leningrad, 1979/1980), Lecture Notes in Math. 864, Springer, Berlin, 1981, 1-89.

[2] T. Aoki, Locally bounded linear topological spaces, Proc. Imp. Acad. Tokyo (1942), no. 10.

[3] N. Asmar and S. J. Montgomery-Smith, On the distribution of Sidon series, Ark. Mat. 31 (1993), 13-26.

[4] G. Bennett, Lectures on matrix transformations of $l^{p}$ spaces, in: Notes in Banach Spaces, Univ. Texas Press, Austin, Tex., 1980, 39-80.

[5] A. Bonami, Ensembles $\Lambda(p)$ dans le dual de $D^{\infty}$, Ann. Inst. Fourier (Grenoble) 18 (1968), 193-204 (1969).

[6] J. Bourgain and L. Tzafriri, Invertibility of "large" submatrices with applications to the geometry of Banach spaces and harmonic analysis, Israel J. Math. 57 (1987), 137-224.

[7] V. H. de la Peña and E. Giné, Decoupling, Probability and its Applications (New York), Springer-Verlag, New York, 1999.

[8] V. H. de la Peña and S. J. Montgomery-Smith, Bounds on the tail probability of U-statistics and quadratic forms, Bull. Amer. Math. Soc. (N.S.) 31 (1994), 223-227.

[9] —, 一, Decoupling inequalities for the tail probabilities of multivariate U-statistics, Ann. Probab. 23 (1995), 806-816.

[10] A. Grothendieck, Résumé de la théorie métrique des produits tensoriels topologiques, Bol. Soc. Mat. São Paulo 8 (1953).

[11] S. Hartman and C. Ryll-Nardzewski, Almost periodic extensions of functions, Colloq. Math. 12 (1964), 23-39. 
[12] J.-P. Kahane, Ensembles de Ryll-Nardzewski et ensembles de Helson, Colloq. Math. 15 (1966), 87-92.

[13] —, Some Random Series of Functions, Heath, Lexington, Mass., 1968.

[14] N. J. Kalton, The three space problem for locally bounded F-spaces, Compositio Math. 37 (1978), 243-276.

[15] N. J. Kalton, Convexity, type and the three space problem, Studia Math. 69 (1980/81), $247-287$.

[16] -, Convexity conditions for nonlocally convex lattices, Glasgow Math. J. 25 (1984), 141-152.

[17] —, Banach envelopes of nonlocally convex spaces, Canad. J. Math. 38 (1986), 65-86.

[18] —, Plurisubharmonic functions on quasi-Banach spaces, Studia Math. 84 (1986), 297-324.

[19] —, On vector-valued inequalities for Sidon sets and sets of interpolation, Colloq. Math. 64 (1993), 233-244.

[20] —, Rademacher series and decoupling (to appear).

[21] N. J. Kalton and S.-C. Tam, Factorization theorems for quasi-normed spaces, Houston J. Math. 19 (1993), 301-317.

[22] S. V. Kislyakov, On BMO-regular lattices of measurable functions, Algebra i Analiz 14 (2002), 117-135 (in Russian).

[23] S. Kwapien, Isomorphic characterizations of inner product spaces by orthogonal series with vector valued coefficients, Studia Math. 44 (1972), 583-595.

[24] —, Decoupling inequalities for polynomial chaos, Ann. Probab. 15 (1987), 1062-1071.

[25] J. Lindenstrauss and A. Pełczyński, Absolutely summing operators in $L_{p}$-spaces and their applications, Studia Math. 29 (1968), 275-326.

[26] J. Marcinkiewicz and A. Zygmund, Quelques inégalités pour les opérations linéaires, Fund. Math. (1939), 112-121.

[27] B. Maurey, Théorèmes de factorisation pour les opérateurs linéaires à valeurs dans les espaces $L^{p}$, Astérisque 11 (1974).

[28] - Type, cotype and K-convexity, in: Handbook of the Geometry of Banach Spaces, Vol. 2, North-Holland, Amsterdam, 2003, 1299-1332.

[29] J. F. Méla, Sur les ensembles d'interpolation de C. Ryll-Nardzewski et de S. Hartman, Studia Math. 29 (1967/1968), 167-193.

[30] E. M. Nikišin, A resonance theorem and series in eigenfunctions of the Laplace operator, Izv. Akad. Nauk SSSR Ser. Mat. 36 (1972), 795-813 (in Russian).

[31] G. Nordlander, On sign-independent and almost sign-independent convergence in normed linear spaces, Ark. Mat. 4 (1962), 287-296 (1962).

[32] W. Orlicz, Über unbedingte Konvergenz in Funktionenraümen I, Studia Math. 4 (1933), $33-37$.

[33] —, Über unbedingte Konvergenz in Funktionenraümen II, Studia Math. 4 (1933), 41-47.

[34] R. E. A. C. Paley, A remarkable series of orthogonal functions, Proc. London Math. Soc. 34 (1932), 241-264.

[35] A. Pełczyński, Commensurate sequences of characters, Proc. Amer. Math. Soc. 104 (1988), $525-531$.

[36] G. Pisier, Les inégalités de Khintchine-Kahane, d'après C. Borell, in: Séminaire Sur la Géométrie Des Espaces de Banach (1977-1978), École Polytech., Palaiseau, 1978, Exp. No. 7.

[37] —, Un théorème sur les opérateurs linéaires entre espaces de Banach qui se factorisent par un espace de Hilbert, Ann. Sci. École Norm. Sup. (4) 13 (1980), 23-43.

[38] —, Counterexamples to a conjecture of Grothendieck, Acta Math. 151 (1983), 181-208. 
[39] G. Pisier, Factorization of Linear Operators and Geometry of Banach Spaces, CBMS Regional Conf. Ser. Math. 60, Washington, DC, 1986.

[40] - Factorization of operators through $L_{p \infty}$ or $L_{p 1}$ and noncommutative generalizations, Math. Ann. 276 (1986), 105-136.

[41] —, A simple proof of a theorem of Jean Bourgain, Michigan Math. J. 39 (1992), 475-484.

[42] S. Rolewicz, On a certain class of linear metric spaces, Bull. Acad. Polon. Sci. Cl. III 5 (1957), 471-473. 